\title{
The Quality of Life and Economic Burden of Erectile Dysfunction
}

This article was published in the following Dove Press journal:

Research and Reports in Urology

\author{
Dean S Elterman' \\ Samir K Bhattacharyya ${ }^{2}$ \\ Michael Mafilios iD ${ }^{3}$ \\ Emily Woodward ${ }^{2}$ \\ Krista Nitschelm iD ${ }^{2}$ \\ Arthur L Burnett ${ }^{4}$ \\ 'University of Toronto, Toronto, ON, \\ Canada; ${ }^{2}$ Boston Scientific Corporation, \\ Marlborough, MA, USA; ${ }^{3}$ Health \\ Economics Associates, San Diego, CA, \\ USA; ${ }^{4}$ The Johns Hopkins University \\ School of Medicine, Baltimore, MD, USA
}

Correspondence: Michael Mafilios

Health Economics Associates, 1804

Garnet Avenue, Ste 4I2, San Diego, CA,

92109 , USA

$\mathrm{Tel}+\mid$ 858-367-9|40

Email info@hea-consulting.com

\begin{abstract}
Erectile dysfunction (ED) is a common disorder in adult males that results in withdrawal from sexual intimacy, psychosocial problems (ie, poor self-esteem, depression, anxiety), decreased work productivity, and reduction in quality of life for both the men suffering from ED and their female partners. A pragmatic literature review was undertaken using PUBMED to identify original research studies published over the past 20 years that assessed the impact of ED on a male's quality of life, the impact of ED on a female partner's quality of life, or the economic impact of ED on employers. Twenty studies were selected for inclusion. This review showed that men with ED have a poorer quality of life than men without ED ( $\mathrm{n}=9$ studies). Results from a global burden of illness study showed that men with ED report substantially lower SF-36 Mental and Physical Component Summary scores and SF-6D scores compared to men without ED $(p<0.001)$. Similarly, the partner is also negatively impacted by ED due to relationship difficulties and decreased sexual satisfaction ( $n=8$ studies). Results from the Female Experience of Men's Attitudes to Life Events and Sexuality study showed that females were significantly less satisfied and engaged in sexual activity less frequently after their partner developed ED $(p<0.001)$. ED also poses a substantial economic burden on employers ( $n=3$ studies). An observational study in men aged 40-70 showed that men with ED had significantly higher rates of absenteeism (2x) and work productivity impairment compared to men without ED $(p<0.001)$. Overall, this contemporary review demonstrated that ED imposes a substantial quality of life burden on men and their female partners as well as a significant economic burden on their employers. These findings underscore the need for more education and awareness of the burden of ED and greater access to appropriate ED treatments to help alleviate this burden.
\end{abstract}

Keywords: erectile dysfunction, work productivity, absenteeism, burden, quality of life, economics, sexual partners, sexual dysfunction

\section{Introduction}

Erectile dysfunction (ED) is characterized by the inability to achieve or maintain an erection sufficient for satisfactory sexual performance. ${ }^{1}$ ED is a common disorder of sexual function in adult males. The overall prevalence of ED in men aged 20 years or older in the US is $18.4 \%$ suggesting that ED affects approximately 18 million men. ${ }^{2}$ Globally, ED prevalence estimates reported in the published literature have ranged from $10 \%$ to $48 \%$ depending on the various study methodologies. $^{3-6}$ The prevalence of ED differs remarkably by age. Data from the US National Health and Nutrition Examination Survey (NHANES) indicates that the prevalence of ED increases from about 5\% in men aged 20-39 years, to $14.8 \%$ in men aged $40-59$, and to $70 \%$ in men aged 70 years and older. ${ }^{2}$ Common 
causes of ED include psychological conditions (ie, depression, anxiety, and stress), neurologic conditions (ie, stroke, Alzheimer's disease, spinal cord injury), hormonal conditions, prostate conditions (ie, radiotherapy and/or surgery for prostate cancer), and cardiovascular conditions. ${ }^{7}$ ED may also be induced by medications for managing other chronic conditions (ie, antidepressants, antihistamines, antihypertensives) or lifestyle factors (ie, alcohol or substance abuse, obesity). ${ }^{7}$ The 2018 American Urological Association (AUA) clinical guidelines for ED recommend a shared decision-making approach between the physician and patient for the various treatment options ranging from oral prescription medications such as phosphodiesterase type 5 (PDE5) inhibitors, to vacuum erection devices, selfadministered intracavernous injections, intraurethral suppositories, and penile prosthesis implantation. ${ }^{8}$ Despite the high prevalence of ED and its association with many healthcare conditions, ED remains an underdiagnosed and undertreated condition. ${ }^{9-11}$

ED is not a life-threatening condition. However, ED results in withdrawal from sexual intimacy, reduced quality of life, and decreased work productivity. ${ }^{12}$ This condition is associated with many psychosocial problems such as anxiety, depression, anger, frustration, poor self-esteem, guilt, lack of confidence, and limited intimacy. ${ }^{13}$ A male's partner may also be negatively impacted by ED due to relationship difficulties and sexual dissatisfaction. ${ }^{14,15} \mathrm{ED}$ also negatively affects employers as men with ED have higher rates of absenteeism due to psychosocial and physical reasons as well as work productivity impairment than men without ED. ${ }^{3}$

With men and their female partners increasingly seeking to preserve sexual function and quality of life as they age, it is important to understand the current research assessing the humanistic impact of ED. Additionally, since ED may impair work productivity, it is important to evaluate the economic impact of ED on an employer. The primary objectives of this pragmatic literature review are to characterize the impact of ED on a male's quality of life, the impact of ED on a female partner's quality of life, and the economic impact of ED on an employer.

\section{Methods}

A literature search was undertaken in PUBMED to identify original research studies published from January 2000 through May 2020 that assessed (1) the impact of ED on a male's quality of life, (2) the impact of ED on a female partner's quality of life, or (3) the economic impact of ED on an employer. Non-systematic review studies, case studies, editorials, letters, or commentaries were excluded.

Although a 20-year time frame was selected for the literature search, the review focused on the most contemporary studies published on the quality of life burden or economic burden of ED. Each search was conducted using controlled vocabulary and limited to studies published in English and involving humans. The preliminary literature searches identified 1635 potentially relevant studies (Figure 1). All of the abstracts from the literature searches were reviewed for potential inclusion in the review. Additional studies were identified based on a free text search of the internet and a review of the reference lists from the full-text studies. Studies that presented the comparative burden in participants with ED versus participants without ED were highlighted. Studies reporting exclusively on the clinical burden of ED or epidemiological burden of ED (ie, etiology, incidence and/or prevalence) were beyond the scope of this research. Additionally, an examination of studies exploring the relative risk or association between ED and other disorders such as diabetes, cardiovascular disease, prostatectomy following prostate cancer, hypertension, obesity, or lower urinary tract symptoms were beyond the scope of this research. Finally, studies were excluded if they only reported on the economic outcomes or quality of life outcomes associated with specific ED treatments and did not contain any information on the burden of ED. A total of 20 studies were selected for inclusion in this review (Figure 1).

\section{Quality of Life Burden of ED Impact of ED on a Male's Quality of Life} Quality of life is defined as a person's subjective perception of their own physical, social, or emotional function and overall well-being in relation to their health. Many US and non-US studies $(n=9)$ have shown that men with ED have a poorer quality of life than men without ED. ${ }^{3,9,13,16-21}$ A global burden of illness study (2019) examined the impact of ED on quality of life in men aged 40-70 years from eight countries (Brazil, China, France, Germany, Italy, Spain, the United Kingdom, and the United States). ${ }^{3}$ This cross-sectional observational study analyzed data collected from 52,697 adult men using the 2015-2016 National Health and Wellness Survey ( $n=26,192$ with ED, $n=25,505$ without ED). The Short-Form 36-Item Health Survey (SF-36) and the ShortForm 6-Dimension Health Survey (SF-6D) were used to 


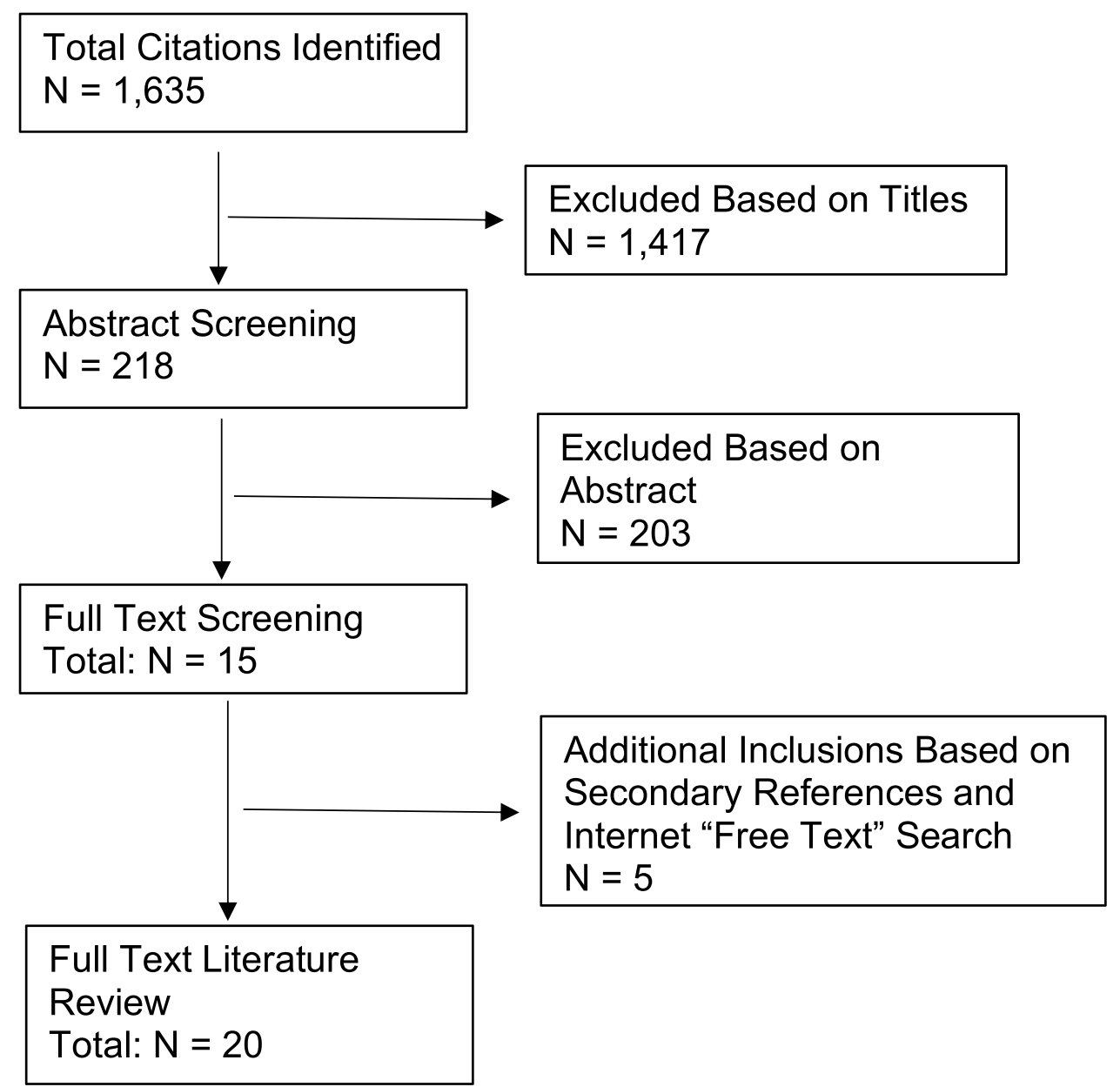

Figure I PRISMA flow diagram of study identification and selection.

Notes: PRISMA figure adapted from Liberati A, Altman D, Tetzlaff J, et al. The PRISMA statement for reporting systematic reviews and meta-analyses of studies that evaluate health care interventions: explanation and elaboration. Journal of clinical epidemiology. 2009;62(10). Creative Commons. ${ }^{30}$

Abbreviation: PRISMA, Preferred Reporting Items for Systematic Reviews and Meta-Analyses.

assess the general quality of life in this population. SF-36 scores range from 0 (worse health) to 100 (better health), with higher scores indicative of better quality of life. The SF-36 is the most frequently used generic quality of life assessment tool in ED studies since it addresses multidimensional domains of quality of life relevant to ED such as mental health, physical functioning, bodily pain, emotional health, commonly performed daily activities, and social functioning. The SF-6D is a generic measure of health outcome that has been used in ED studies to derive health state utilities. A utility is a measure of the quality of life ranging on an interval scale between 0 for death and 1 for complete health, with higher scores indicative of better quality of life. The results of the study showed that men with ED had lower SF-36 mental component summary scores (MCS) and physical component summary scores (PCS) than men without ED (MCS: 46.7 vs 51.2, $\mathrm{p}<0.001$; PCS: 48.3 vs 53.0, $\mathrm{p}<0.001)$ (Table 1$){ }^{3}$ Additionally, this study showed the men with ED had lower SF-6D health state utility scores than men without ED (SF-6D: 0.69 vs $0.78, \mathrm{p}<0.001$ ). For the sub-group analysis of US men, the study showed that US men with ED had lower SF-36 mental component summary scores and physical component summary scores than US men without ED (MCS: 48.5 vs 52; PCS: 49.1 vs 51.9). ${ }^{3}$ After an adjustment for potential cofounding variables, this study showed that the differences in quality of life scores between men with ED and those without ED exceeded the minimally important difference for the SF36 Mental Component Summary score (ie, 3.0 points) and the SF-6D score (ie, 0.041 points). ${ }^{3}$

A European burden of illness study (2014) examined the impact of ED on quality of life in men with ED from five European nations (France, Germany, Italy, Spain, and 
Table I Quality of Life Burden in Respondents with ED Compared to without ED

\begin{tabular}{|c|c|c|c|}
\hline Publication & Quality of Life Survey & Respondents with ED & Respondents without ED \\
\hline \multirow[t]{2}{*}{ Goldstein $(2019)^{3}$} & SF-36 Mental Component Summary & 46.7 & 51.2 \\
\hline & SF-36 Physical Component Summary & 48.3 & 53.0 \\
\hline \multirow[t]{2}{*}{ Jannini $(2014)^{9}$} & SF-12 Mental Domain (Ages 40-59 cohort) & 42.9 & 48.3 \\
\hline & SF-12 Physical Domain (Ages 40-59 cohort) & 44.4 & 59.6 \\
\hline \multirow[t]{2}{*}{ Sánchez-Cruz $(2003)^{18}$} & SF-36 Mental Component Summary & 48.9 & 52.3 \\
\hline & SF-36 Physical Component Summary & 42.3 & 53.6 \\
\hline Litwin $(1998)^{17}$ & SF-36 Physical Function & 55.7 & $79.9 *$ \\
\hline
\end{tabular}

Note: *Comparator group for age-matched population normals aged 55-64.

the United Kingdom) using data collected from the 2011 National Health and Wellness Survey. ${ }^{9}$ Of the 28,511 survey respondents, 5184 men met the criteria for selfreported ED during the past 6 months. The Short-Form 12Item Health Survey (SF-12) was used to assess the general quality of life in the study population. SF-12 scores range from 0 (worse health) to 100 (better health), with higher scores indicative of better quality of life. The authors examined the SF-12 quality of life scores in three age cohorts: $18-39$ years; $40-59$ years; and $\geq 60$ years. The results of the study showed that men with ED had significantly lower SF-12 mental domain quality of life scores and physical domain quality of life scores than men without ED for all three age cohorts. ${ }^{9}$ For example, in the 40-59 years cohort, men with ED had lower SF-12 mental domain quality of life scores and physical domain quality of life scores than men without ED (SF-12 Mental Domain: 42.9 vs 48.3; Physical Domain: 44.4 vs 59.6) (Table 1).

A real-world observational study (2003) examined the prevalence of ED in Spanish men aged 25 to 70 years and the impact of ED on quality of life. ${ }^{18}$ The SF-36 was used to assess the general quality of life in the study population ( $\mathrm{n}=295$ with ED; $\mathrm{n}=2160$ without ED). The results of the study showed that Spanish men with ED had lower SF-36 mental component summary scores and physical component summary scores than Spanish men without ED (MCS: 48.9 vs $52.3, \mathrm{p}<0.01$; PCS: 42.3 vs 53.6, $\mathrm{p}<0.01)$ (Table 1). ${ }^{18}$

A US study (1998) examined the quality of life in veterans with $\mathrm{ED}(\mathrm{n}=22)$ at a Veterans Affairs Medical Center. ${ }^{17}$ The SF-36 was used to assess the general quality of life in this ED population. The SF-36 scores of the ED population were then compared to quality of life SF-36 scores of the general population (community-based norms for men aged 55-64). The results of the study showed that men with ED had lower emotional, social, and physical functioning compared to aged-matched population norms indicating a profound impairment in quality of life. ${ }^{17}$ All of the respondents with ED scored lower than communitybased population norms for each of the SF-36 domains. ${ }^{17}$ In particular, the respondents scored substantially lower than population norms on the physical domains, consistent with the clinical experience of poor physical health found in populations of veterans.

The quality of life burden from ED is similar to the quality of life burden observed in other medical conditions. In the SF-36 Health Survey Manual \& Interpretation Guide (1993), the authors of the SF-36 Health Survey estimated quality of life norms for the following five medical conditions: hypertension, congestive heart failure, type II diabetes, myocardial infarction, and clinical depression. ${ }^{29}$ The SF-36 scores reported for the General Health SF-36 domain in these five conditions were 63, 47, 56,59 , and 53, respectively, indicating an impairment in quality of life similar to ED. ${ }^{29}$

Depression and anxiety are common complaints among men with ED. ${ }^{13,16,20,21} \mathrm{~A}$ recently published meta-analysis (2018) examining the relationship between ED and depression in six studies $(n=22,527$ participants) revealed that exposure to ED increased the risk of depression by $192 \%$ and that the incidence of depression is 2.92 times higher in men with ED than in those without ED. ${ }^{21}$ Similarly, a meta-analysis (2012) examining the relationship between sexual dysfunction (including ED) and depression in six studies ( $\mathrm{n}=11,171$ participants) demonstrated that sexual dysfunction increased the risk of depression (OR 2.30 $[1.74,3.03]){ }^{20}$ The Massachusetts Male Aging Study $(\mathrm{n}=1709)$, a cross-sectional, community-based random sample survey of health and aging in US men aged 40 to 
70 years found that psychosocial factors such as depression, low levels of dominance, and anger (either expressed outward or directed inward) were strongly associated with ED. ${ }^{16}$

Overall, these studies demonstrate that men with ED suffer a deterioration in psychological, social, and physical well-being compared to those without ED.

\section{Impact of ED on a Female Partner's Quality of Life}

There is a growing body of research that shows ED adversely affects the relationship with a partner due to the negative impact of ED on a partner's personal sexual satisfaction and sexual function ( $\mathrm{n}=8$ studies). ${ }^{14,15,19,22-26}$ The Female Experience of Men's Attitudes to Life Events and Sexuality (FEMALES) study (2005) examined the frequency of sexual activity and the nature of the sexual experience in female partners of men with ED $(n=293)$ both before and after the development of their partner's ED. ${ }^{14}$ The females participating in this study were from seven countries: Brazil, France, Germany, Italy, Spain, the United Kingdom, and the United States. A questionnaire was developed to reflect the female partner's perspective comprising 65 items relating to the woman's sexual experience and level of sexual satisfaction before and after her partner developed ED. Additionally, the woman's degree of satisfaction with their sexual relationship was measured using a five-point scale ranging from very satisfied to very dissatisfied before and after their partner developed ED. The results of this study showed that the number of females who had previously felt sexual desire, arousal, or achieved orgasm "almost always" or "most times" during sexual activity was significantly reduced after their partners developed ED. ${ }^{14}$ Additionally, this study showed that females had decreased satisfaction in the sexual relationship and engaged in sexual activity significantly less frequently after their partner developed ED. $^{14}$

A cross-sectional study (2013) investigated the association between female sexual function and the male partner's ED in 2159 females in Taiwan. ${ }^{24}$ Female sexual function and male erectile function were assessed by the Female Sexual Function Index (FSFI) and by the International Index of Erectile Function. The results of the study showed that a partner's ED was a significant risk factor for female sexual difficulties including problems with arousal, orgasm, sexual satisfaction, and sexual pain (OR
2.5-3.3, $\mathrm{p}<0.01) .{ }^{24}$ Female partners of men with ED had a significantly higher prevalence of sexual difficulty in every FSFI domain than female partners of men without ED (overall sexual difficulty: $71.8 \%$ vs $38.1 \%$; desire difficulty: $61.5 \%$ vs $41.8 \%$; arousal difficulty: $36.7 \%$ vs $13.1 \%$; orgasm difficulty: $17.4 \%$ vs $6.9 \%$; pain difficulty: $17.4 \%$ vs $7.7 \%)^{24}$

A prospective study (2004) assessing the sexual function and sexual satisfaction in Turkish females whose male partners had ED $(n=38)$ compared to females whose male partners did not have ED ( $n=49$; control group) found that female partners of men with ED had significantly lower levels of sexual satisfaction $(\mathrm{p}<0.001)$, sexual arousal $(\mathrm{p}=0.009)$, and orgasm $(\mathrm{p}=0.006)$ than females in the control group. ${ }^{22}$ Similarly, a prospective survey study (2005) assessing sexual dysfunction in 113 female partners of men with ED in Israel found that $55 \%$ of females in the study experienced sexual dysfunction due to their partner's ED. ${ }^{23}$ A survey study (2000) in 1335 females and 1475 males from Sweden reported that $69 \%$ of males who had experienced ED and $74 \%$ of females with an ED-inflicted male partner stated that ED was a "problem" in their relationship. ${ }^{19}$ The study also showed that $82 \%$ of female partners of ED-inflicted men were sexually dissatisfied. ${ }^{19}$

Overall, although ED is a condition that physically impacts males, these studies show that ED results in several negative aspects on the quality of life for each partner in a sexual relationship.

\section{Economic Burden of ED Impact of ED on Work Productivity}

ED can impose a substantial economic impact on an employer ( $n=3$ studies). ${ }^{3,9,27}$ Men with ED suffer from higher rates of absenteeism, presenteeism (impairment while present at work), and work productivity loss than men without ED. ${ }^{3,9}$ A global burden of illness study (2019) examined work productivity and activity impairment among men with ED from Brazil, China, France, Germany, Italy, Spain, the United Kingdom, and the United States. ${ }^{3}$ This cross-sectional observational study analyzed data collected from 52,697 adult men aged 40-70 years-old using the 2015-2016 National Health and Wellness Survey. ${ }^{3}$ Employment productivity was assessed using the Work Productivity and Impairment General Health (WPAI-GH) questionnaire. $^{3}$ Work productivity outcomes assessed included absenteeism (defined as the percentage of work time missed because of one's health in the past 7 days), 
presenteeism (defined as the percentage of impairment experienced while at work in the past 7 days because of one's health), and overall work productivity loss (overall work impairment measured by combining absenteeism and presenteeism to determine the total percentage of missed time). ${ }^{3}$ The analyses controlled for potential confounders including common ED comorbidities (ie, hypertension, dyslipidemia, diabetes, and depression) and unhealthy lifestyle factors (ie, smoking, alcohol abuse, and lack of exercise). This study found that men with ED reported significantly higher rates of absenteeism $(7.1 \%$ vs $3.2 \%, \mathrm{p}<0.001)$, impairment while present $(22.5 \%$ vs $10.1 \%, \mathrm{p}<0.001)$, and overall work productivity impairment $(24.8 \%$ vs $11.2 \%$, $\mathrm{p}<0.001$ ) than men without ED (Table 2). ${ }^{3}$ These results demonstrate that men with ED experience more than twice as much impairment in overall work productivity compared to men without $\mathrm{ED}(\mathrm{p}<0.05)$. Among the different countries, men from France with ED (vs without ED) reported the highest rate of absenteeism (9.4\% vs 5.7\%) while men from Italy with ED reported the highest rate of overall work impairment $(32.5 \%$ vs $16.1 \%){ }^{3}$ For US respondents, the study found that men with ED compared to men without ED reported higher rates of absenteeism (4.7\% vs $1.9 \%$ ), impairment while present (19\% vs $8.9 \%$ ), and work productivity impairment $(20.5 \%$ vs $9.7 \%) .{ }^{3}$ These findings show that men with ED in the US have approximately 2.11 times as much overall work productivity impairment as men without ED $(\mathrm{p}<0.05){ }^{3}$

A European burden of illness study (2014) examined work productivity impairment and the unmet needs of men with ED from five European nations (France, Germany, Italy, Spain, and the United Kingdom). ${ }^{9}$ This study analyzed data collected from the 2011 National Health and Wellness Survey on a population of 28,511 men and was focused on the sub-group of men who self-reported ED in the past 6 months $(n=5184){ }^{9}$ The authors examined work productivity outcomes in three age cohorts: 18-39 years (without $\mathrm{ED}=88750$ vs $\mathrm{ED}=508$ ); 40-59 years (without
$\mathrm{ED}=83,432$ vs $\mathrm{ED}=1736$ ); and $\geq 60$ years (without $\mathrm{ED}=5090$ vs $\mathrm{ED}=2940$ ). Employment productivity was assessed using the WPAI-GH questionnaire. The work productivity outcomes assessed included absenteeism, presenteeism, and overall work productivity loss. ${ }^{9}$ This study found that European men with ED aged 18-39 years had higher rates of absenteeism (11.6\% vs 5.0\%), impairment while present $(30.3 \%$ vs $15.8 \%)$, and overall work productivity loss (35.4\% vs 18.9\%) compared to European men aged 18-39 years without ED. ${ }^{9}$ Similarly, the results of the study showed that European men with ED aged 40-59 years also had significantly higher rates of absenteeism (6.9\% vs $4.4 \%)$, impairment while present $(19.4 \%$ vs $13.4 \%)$, and overall work productivity loss $(23.9 \%$ vs $16.5 \%$ ) compared to European men aged 40-59 years without ED (Table 2). ${ }^{9}$ The findings were similar for men with ED over the age of 60 . Overall, these findings show that European men with ED have significantly higher work impairment than men without ED across all agerange categories. ${ }^{9}$ A limitation of this study is that potential confounders such as comorbid illnesses associated with ED were not controlled for in the analysis. The findings from these burden of illness studies ${ }^{3,9}$ are consistent with previous studies showing an impairment in work productivity in men with ED. ${ }^{13,27}$

\section{Undertreatment of ED}

Although many men consider ED a serious health issue that impairs their quality of life, there is a subpopulation of men who do not consider ED to be a serious problem, are too embarrassed to seek help from a physician about their sexual health, or do not have access to ED therapies. ${ }^{9}$ For example, an Asian survey study (2005) of sexual behavior and sexual dysfunction in adult men aged $40-80$ years in the urban population of Asian countries found that $45 \%$ of men with sexual dysfunction did not seek help or advice for their condition and only $21 \%$ sought medical care. ${ }^{28}$ The main reasons cited by these men for not consulting

Table 2 Economic Impact of ED on Work Productivity in Respondents with ED Compared to without ED

\begin{tabular}{|l|l|c|c|}
\hline Publication & Work Productivity Domain & Respondents with ED & Respondents without ED \\
\hline Goldstein (2019) & Absenteeism & $7.1 \%$ & $3.2 \%$ \\
& Presenteeism & $22.5 \%$ & $10.1 \%$ \\
& Work Productivity Impairment & $24.8 \%$ & $11.2 \%$ \\
\hline \multirow{2}{*}{ Jannini (2014) } & Absenteeism (Ages 40-59 cohort) & $6.9 \%$ & $4.4 \%$ \\
& Presenteeism (Ages 40-59 cohort) & $19.4 \%$ & $13.4 \%$ \\
& Work Productivity Loss (Ages 40-59 cohort) & $23.9 \%$ & $16.5 \%$ \\
\hline
\end{tabular}


a doctor about their condition included the belief that their condition was not a medical issue, embarrassment, and access to or affordability of medical care for their sexual dysfunction. $^{28}$

The European burden of illness study (2014) discussed above examined the impact of undertreatment of ED on work productivity outcomes in a sub-set of respondents with severe ED. ${ }^{9}$ The results of this subanalysis found that European men with severe ED who were not treated for ED $(n=837)$ also had high rates of absenteeism, presenteeism, and work productivity loss $\left(6.5 \%, 18 \%\right.$, and $24.8 \%$, respectively). ${ }^{9}$ The results of this study also show that more than half of European men with self-reported ED (52\%) did not discuss their condition with their physician. ${ }^{9}$ Among the sub-group of men with ED who had consulted their physician, only $32 \%$ were receiving an ED medication (PDE5 inhibitor). ${ }^{9}$ Overall, despite the high prevalence of ED and the negative impact of ED on quality of life and work productivity, ED remains an undertreated condition. $^{9,10,28}$

\section{Conclusion}

This pragmatic literature review demonstrates that ED imposes a substantial quality of life burden on men and their female partners as well as a significant economic burden on their employers. Several real-world studies have demonstrated that men with ED have a poorer quality of life than men without $\mathrm{ED}$, regardless of age. Similarly, female partners of men with ED are also negatively impacted by ED due to relationship difficulties and decreased relationship satisfaction. Men with ED at any age impose a substantial economic burden on employers due to higher rates of absenteeism, presenteeism (impairment while present at work), and work productivity loss compared to men without ED. Although ED is not a life-threatening disease, the findings from this review suggest there is a need for better management and access to appropriate ED treatments to help alleviate the substantial quality of life and economic burden of this condition. These findings also underscore the need for more education on the etiology of ED, more understanding of the available treatment options, and more awareness of the physical and emotional burden ED can impose on men and their partners.

\section{Acknowledgment}

The abstract and interim findings of this paper were presented virtually at the 2020 Annual Meeting of the International Society for Pharmacoeconomics and Outcomes Research in Europe (ISPOR Europe). November 16-19, 2020. The poster's abstract was published in Value in Health 2020; 23:Supplement 2: S540S541. DOI:https://doi.org/10.1016/j.jval.2020.08.810.

\section{Disclosure}

Dr. Dean Elterman: Clinical consultant for Boston Scientific; Dr. Samir Bhattacharyya: Employee of Boston Scientific Corporation; Michael Mafilios: Employee of Health Economics Associates which was contracted to conduct the literature review; Emily Woodward: Employee of Boston Scientific Corporation; Krista Nitschelm: Employee of Boston Scientific Corporation; Dr. Arthur Burnett: Reports grants from Boston Scientific, Andrology (journal), Astellas, Comphya, Futura Medical, International Urology and Nephrology (journal), Lilly LLC, Myriad Genetics, National Institutes of Health, New England Research Institute, Novartis Pharmaceuticals, Pfizer, Reflexonic LLC, UroMissionsWorks Inc., Urology Times Editorial Council, and personal fees from MHN Biotec, during the conduct of the study.

\section{References}

1. National Institutes of Health. Impotence. NIH consensus statement; 1992. Available from: https://consensus.nih.gov/1992/1992impoten ce091html.htm. Accessed August 15, 2020.

2. Selvin E, Burnett AL, Platz EA. Prevalence and risk factors for erectile dysfunction in the US. Am J Med. 2007;120(2):151-157. doi:10.1016/ j.amjmed.2006.06.010

3. Goldstein I, Goren A, Li VW, Maculaitis MC, Tang WY, Hassan TA. The association of erectile dysfunction with productivity and absenteeism in eight countries globally. Int J Clin Pract. 2019;73(11):e13384. doi:10.1111/ijcp. 13384

4. Mulhall J, King R, Glina S, Hvidsten K. Importance of and satisfaction with sex among men and women worldwide: results of the global better sex survey. J Sex Med. 2008;5(4):788-795. doi:10.1111/j.17436109.2007.00765.x

5. Nicolosi A, Laumann EO, Glasser DB, et al. Sexual behavior and sexual dysfunctions after age 40: the global study of sexual attitudes and behaviors. Urology. 2004;64(5):991-997. doi:10.1016/j.urology. 2004.06.055

6. Kessler A, Sollie S, Challacombe B, Briggs K, Van Hemelrijck M. The global prevalence of erectile dysfunction: a review. BJU Int. 2019;2.

7. Shindel AW, Brandt WO, Bochinski D, et al. Medical and surgical therapy of erectile dysfunction. In: Feingold KR, Anawalt B, Boyce A, et al. editors. Endotext. South Dartmouth (MA): MDText.com, Inc.; 2000. Available from: https://www.ncbi.nlm.nih.gov/books/ NBK278925/. Accessed August 15, 2020. 
8. Burnett AL, Nehra A, Breau RH, et al. Erectile dysfunction: AUA guideline. J Urol. 2018;200(3):633-641. doi:10.1016/j.juro.2018.05. 004

9. Jannini EA, Sternbach N, Limoncin E, et al. Health-related characteristics and unmet needs of men with erectile dysfunction: a survey in five European countries. J Sex Med. 2014;11(1):40-50. doi:10. $1111 /$ jsm. 12344

10. Frost M, Wraae K, Gudex C, et al. Chronic diseases in elderly men: under-reporting and under-diagnosis. Age Ageing. 2012;41(2): 177-183. doi:10.1093/ageing/afr153

11. Goldstein I, Goren A, Li VW, Tang WY, Hassan TA. Epidemiology update of erectile dysfunction in eight countries with high burden. Sex Med Rev. 2020;8(1):48-58. doi:10.1016/j.sxmr.2019.06.008

12. Wessells H, Joyce G, Wise M, Wilt T. Erectile dysfunction and peyronie's disease. In: Urologic Diseases in America. US Department of Health and Human Services, Public Health Service, National Institutes of Health, National Institute of Diabetes and Digestive and Kidney Diseases. Washington, DC: US Government Printing Office; 2007:485-528.

13. Guest JF, Gupta RD. Health-related quality of life in a UK-based population of men with erectile dysfunction. Pharmacoeconomics. 2002;20(2):109-117. doi:10.2165/00019053-200220020-00004

14. Fisher WA, Rosen RC, Eardley I, Sand M, Goldstein I. Sexual experience of female partners of men with erectile dysfunction: the female experience of men's attitudes to life events and sexuality (FEMALES) study. J Sex Med. 2005;2(5):675-684. doi:10.1111/ j.1743-6109.2005.00118.x

15. Li H, Gao T, Wang R. The role of the sexual partner in managing erectile dysfunction. Nat Rev Urol. 2016;13(3):168-177. doi:10. 1038/nrurol.2015.315

16. Feldman HA, Goldstein I, Hatzichristou DG, Krane RJ, McKinlay JB. Impotence and its medical and psychosocial correlates: results of the Massachusetts male aging study. J Urol. 1994;151 (1):54-61. doi:10.1016/S0022-5347(17)34871-1

17. Litwin MS, Nied RJ, Dhanani N. Health-related quality of life in men with erectile dysfunction. J Gen Intern Med. 1998;13(3):159-166. doi:10.1046/j.1525-1497.1998.00050.x

18. Sánchez-Cruz JJ, Cabrera-León A, Martín-Morales A, Fernández A, Burgos R, Rejas J. Male erectile dysfunction and health-related quality of life. Eur Urol. 2003;44(2):245-253. doi:10.1016/S03022838(03)00215-X

19. Wagner G, Fugl-Meyer KS, Fugl-Meyer AR. Impact of erectile dysfunction on quality of life: patient and partner perspectives. Int J Impot Res. 2000;12(Suppl 4):S144-S146. doi:10.1038/sj.ijir.390 0594
20. Atlantis E, Sullivan T. Bidirectional association between depression and sexual dysfunction: a systematic review and meta-analysis. J Sex Med. 2012;9(6):1497-1507. doi:10.1111/j.1743-6109.2012.02709.x

21. Liu Q, Zhang Y, Wang J, et al. Erectile dysfunction and depression: a systematic review and meta-analysis. J Sex Med. 2018;15 (8):1073-1082. doi:10.1016/j.jsxm.2018.05.016

22. Cayan S, Bozlu M, Canpolat B, Akbay E. The assessment of sexual functions in women with male partners complaining of erectile dysfunction: does treatment of male sexual dysfunction improve female partner's sexual functions? J Sex Marital Ther. 2004;30(5):333-341. doi:10.1080/00926230490465091

23. Greenstein A, Abramov L, Matzkin H, Chen J. Sexual dysfunction in women partners of men with erectile dysfunction. Int J Impot Res. 2006;18(1):44-46. doi:10.1038/sj.ijir.3901367

24. Jiann $\mathrm{BP}, \mathrm{Su} \mathrm{CC}$, Tsai JY. Is female sexual function related to the male partners' erectile function? J Sex Med. 2013;10(2):420-429. doi:10.1111/j.1743-6109.2012.03007.x

25. Riley A, Riley E. Behavioral and clinical findings in couples where the man presents with erectile disorder: a retrospective study. Int J Clin Pract. 2000;54(4):220-224.

26. Zhang H, Fan S, Yip P. The association between female sexual dysfunction and the husband's erectile dysfunction: evidence from married couples in Hong Kong. J Sex Marital Ther. 2016;42 (3):214-222. doi:10.1080/0092623X.2014.996931

27. Plumb JM, Guest JF. Annual cost of erectile dysfunction to UK society. Pharmacoeconomics. 1999;16(6):699-709. doi:10.2165/ 00019053-199916060-00008

28. Nicolosi A, Glasser DB, Kim SC, Marumo K, Laumann EO, GSSAB Investigators' Group. Sexual behavior and dysfunction and helpseeking patterns in adults aged 40-80 years in the urban population of Asian countries. BJU Int. 2005;95(4):609-614. doi:10.1111/j.14 64-410X.2005.05348.x

29. Ware JE, Snow KK, Kosinski M, Gandek B. SF-36 Health Survey: Manual and Interpretation Guide. 1st ed. Boston, MA: The Health Institute, New England Medical Center; 1993:10:22-10:26.

30. Liberati A, Altman D, Tetzlaff J, et al. The PRISMA statement for reporting systematic reviews and meta-analyses of studies that evaluate health care interventions: explanation and elaboration Journal of clinical epidemiology. 2009;62(10).

\section{Publish your work in this journal}

Research and Reports in Urology is an international, peer-reviewed, open access journal publishing original research, reports, editorials, reviews and commentaries on all aspects of adult and pediatric urology in the clinic and laboratory including the following topics: Pathology, pathophysiology of urological disease; Investigation and treatment of urological disease; Pharmacology of drugs used for the treatment of urological disease. The manuscript management system is completely online and includes a very quick and fair peer-review system, which is all easy to use. Visit http://www.dovepress.com/ testimonials.php to read real quotes from published authors. 